\title{
Second International Conference on "Recent Trends in Environment and Sustainable Development" (RTESD 2019)
}

\author{
Rupali Shrivastava $^{1} \cdot$ Rajveer Singh Rajaura ${ }^{2} \cdot$ Subodh Srivastava ${ }^{2} \cdot$ Kumud Kant Awasthi $^{3}$ \\ Published online: 16 January 2021 \\ (C) Springer-Verlag GmbH Germany, part of Springer Nature 2021
}

The environment is getting degraded at a very high speed in India, the reason being rapid growth of population, poverty, urbanization, industrialization, and other related factors. Environmental problems have grown to be severe in many parts of the country, and hence cannot be ignored. The major environmental issues of concern in India are relating to air and water pollution mainly in metropolitan cities and industrial zones. There is a need to encourage participation of academicians and young researchers to overcome the critical environmental, economic, and political challenges being faced by the country and world as a whole. Goals needed to be set for clean water and sanitation; affordable and clean energy; industry innovation and infrastructure with economic growth, sustainable cities, and communities; responsible consumption and production; and maximum action and partnership for the goals. Due to limited resources, there is a fight for utilization leading to their extinction. Unplanned activities are generating a huge amount of waste which go beyond the absorptive capability of the environment. Our effort in the environmental sustainable development conference is to deal with this global environmental crisis, exploring

Responsible Editor: Philippe Garrigues

Rupali Shrivastava

rupali.rec@gmail.com

Rajveer Singh Rajaura

rajveer.nano_cct@live.com

Subodh Srivastava

subodhphy@gmail.com

Kumud Kant Awasthi

kkantawasthi@gmail.com

1 Department of Chemistry, Vivekananda Global University, Jaipur, Rajasthan 303012, India

2 Department of Physics, Vivekananda Global University, Jaipur, Rajasthan 303012, India

3 Department of Life Science, Vivekananda Global University, Jaipur, Rajasthan 303012, India ways and measures that can be undertaken to control the crisis and for remedial purpose.

One who is working on the idea of environmental conservation must understand that sustainable development is only possible and gains real momentum if we are able to conserve resources and use them in a manner that they are sufficiently available for the coming generation as well. Sustainable development is a process that provides for the present generation without compromising on the needs of the future generations. We through a conference have tried to lay emphasis on the idea of passing on an environment with enough and good quality resources to the future generation, just as we have bequeathed from our previous generations. We have to switch to renewable sources of energy as compared to the conventional one that lead to climatic degradation. Increased awareness and consciousness can lead to the resolution of major environmental problems of a country which are related to the level of its economic development. In India, sincere efforts are being made on for the environmental management in a sustainable manner ranging from educating people about environment and its conservation to providing special training for environmental management. Through a conference, we have tried to establish coordination between government agencies, NGOs and the public for the proper management of environment quality and to achieve sustainable development in the country.

The International Conference on "Recent Trends in Environment and Sustainable Development" (RTESD 2019) was a multidisciplinary, peer reviewed international conference on exploration of environment science and engineering-related issues and their possible solutions. This conference provided an opportunity for the researches, academicians, scientists, as well as students to come together and engage in fruitful exchange of views and ideas to pave way for the development of environment and sustainable development.

Publisher's note Springer Nature remains neutral with regard to jurisdictional claims in published maps and institutional affiliations. 


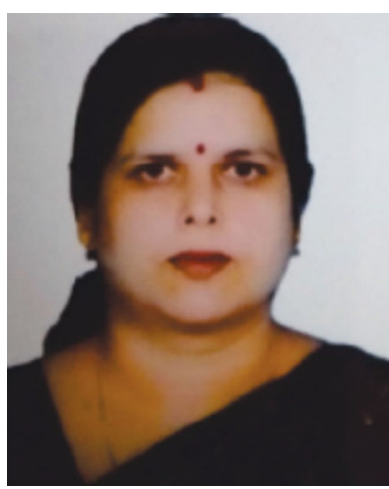

Dr. Rupali Shrivastava is currently an associate provost and professor at the Department of Chemistry, Vivekananda Global University, Jaipur, India. She has completed her MSc Analytical Chemistry in 1999 and Ph.D. in 2004 from Rani Durgavati University Jabalpur, Madhya Pradesh. In her 15 years of teaching carrier, she has engaged graduate and post-graduate level classes of science and engineering students. Her field of research work is techniques like spectroscopy, magnetic studies, thermogravimetric analysis, photoreduction techniques, elemental analysis, pharmaceutical analytical chemistry, environmental chemistry, and photocatalysis. So far, she have published 50 research papers in reputed national and international journals, delivered 20 plenary talks in national and international conferences, and supervised 20 post-graduation level dissertation and $6 \mathrm{Ph}$.D. She is actively involved in conducting conference, seminars, and workshops at national and international level.

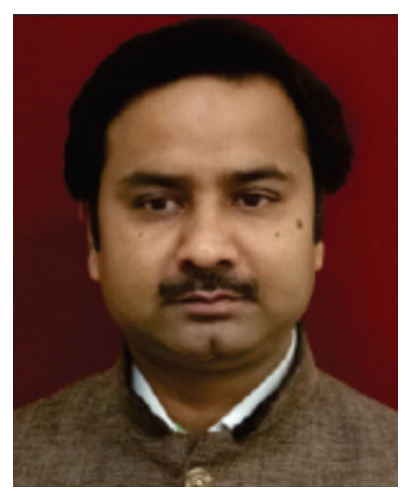

Dr. Subodh Srivastava has completed his post-graduate (M.Sc.) in physics from the Department of Physics, University of Rajasthan, Jaipur, in 2006, followed by Ph.D. in the year 2011 from the University of Rajasthan. His research experience is broadly in the field of renewable energy which includes hydrogen gas sensing, storage, and purification for fuel cell and its application. He has a strong experimental hand and expertise on operating and characterizing units like sputtering coating unit, ellipsometry, and low energy ion irradiation. His research journey include more than 50 research publications of national and international repute and $30+$ presentations in conferences in India and abroad. His research work was appreciated and supported time to time by research grants from funding agencies like DST, UGC, MNRE, and CSIR. His research Zeal was extended further with his research scholars. Besides the research activities, he is an active member of IAPT and INSPIRE education project team. Presently, he is working as an Associate Professor in the Department of Physics, Vivekananda Global University, Jaipur, India.

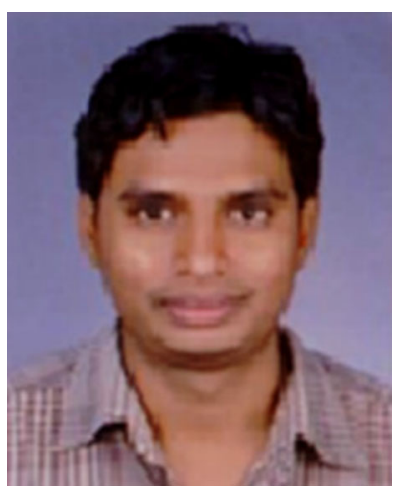

Rajveer Singh Rajaura has completed B. Tech. and M. Tech. in Nanotechnology from centre for converging technologies, University of Rajasthan, Jaipur. His master's dissertation on graphene nanostructure materials for hydrogen storage was carried out at the Inter University Accelerator Centre, New Delhi. He has significant experience in the preparation and characterization of various carbon nanomaterials such as graphene, carbon nanotubes, and polymercarbon nanomaterials nanocomposites. The materials have been used in energy storage and biomedical applications. He has published about 20 papers in peer review journals and 10 presentations in conferences. Mr. Rajaura has served as a reviewer for various international journals including the International Journal of Hydrogen Energy, Materials Research Express, and Nature Scientific Report. He would like to continue working in the field of carbon nanomaterials as an academic interest and also explore the commercialization opportunities.

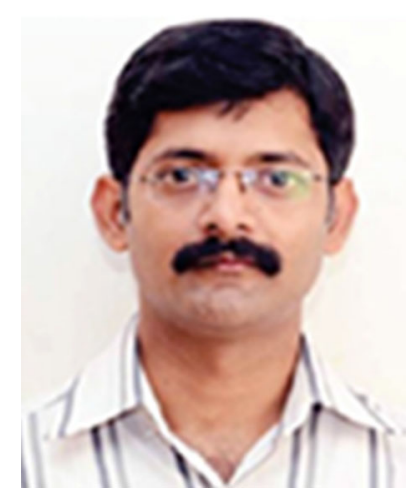

Dr. Kumud Kant Awasthi is currently working as Associate Professor and head of Life Science Department at Vivekananda Global University, Jaipur, Rajasthan, India. He is ex-faculty member of National Institute of Animal Welfare, Faridabad, India. He completed masters and Ph.D. from the University of Rajasthan, Jaipur, India. He has made contribution in the field of nanotoxicology by investigating the toxicity assessment for different nanomaterials in different in vivo and in vitro models and their safe use for drug development. He is an active member of different scientific societies, viz., STOX, ISCA, MRSI, ISLS, ISID, ESTIV, SMRS, and MRS (Singapore), a nominated member of the committee for ethical use of laboratory animals in research activity, a member of editorial boards of journal and organizing committees of national and international conferences, and also a course coordinator. 